\title{
Eurolntervention
}

\section{Final results of a phase Ila, randomised, open-label trial to evaluate the percutaneous intramyocardial transplantation of autologous skeletal myoblasts in congestive heart failure patients: the SEISMIC trial}

Henricus J. Duckers¹, MD, PhD; Jaco Houtgraaf', MD; Christoph Hehrlein², MD, PhD; Joachim Schofer ${ }^{3}$, MD, PhD; Johannes Waltenberger ${ }^{4}$, MD, PhD; Anthony Gershlick ${ }^{5}$, MD, PhD; Jozef Bartunek ${ }^{6}, \mathrm{MD}, \mathrm{PhD}$; Christoph Nienaber ${ }^{7}, \mathrm{MD}$; Carlos Macaya ${ }^{8}, \mathrm{MD}, \mathrm{PhD}$; Nicholas Peters ${ }^{9}$, MD; Peter Smits ${ }^{10}$, MD, PhD; Thomasz Siminiak ${ }^{11}$, MD, PhD; Walter van Mieghem ${ }^{12}$, MD; Victor Legrand ${ }^{13}$, MD, PhD; Patrick W. Serruys ${ }^{1}$, MD, PhD

1. Thoraxcenter, Erasmus University Medical Center, Rotterdam, The Netherlands; 2. Department of Cardiology, University Medical Center of Freiburg, Germany; 3. Universitäres Herzzentrum Hamburg, Germany; 4. Maastricht University Medical Center, The Netherlands; 5. University Hospitals of Leicester, Leicester, United Kingdom, 6. 0.L.V. Ziekenhuis, Aalst, Belgium; 7. Universität-Klinik, Rostock, Germany; 8. Hospital Clínico San Carlos, Ciudad Universitaria, Madrid, Spain; 9. St.Mary's Hospital and Imperial College, London, United Kingdom; 10. Medisch Centrum Rijnmond Zuid, Rotterdam, The Netherlands; 11. University School of Medical Sciences, Poznan, Poland; 12. Z0L Campus St.Jan, Gent, Belgium; 13. UCL Sart Tilman, Liège, Belgium

The authors have no conflict of interest to declare.

\section{KEYWORDS}

Heart failure, cell therapy, percutaneous intervention, skeletal myoblast

\begin{abstract}
Aims: The SEISMIC study was an open-label, prospective, randomised study to assess the safety and feasibility of percutaneous myoblast implantation in heart failure patients with implanted cardioverter-defibrillators (ICD). Methods and results: Patients were randomised 2:1 to autologous skeletal myoblast therapy vs. optimal medical treatment. The primary safety end-point was defined as the incidence of procedural and device related serious adverse events, whereas the efficacy endpoints were defined as the change in global LVEF by MUGA scan, change in NYHA classification of heart failure and in the distance achieved during a sixminute walk test $(6 \mathrm{MW})$ at 6 -month follow-up. Forty subjects were randomised to the treatment arm $(n=26)$, or to the control arm $(n=14)$. There were 12 sustained arrhythmic events and one death after episodes of ventricular tachycardia (VT) in the treatment group and 14 events in the control group (P=ns). At 6-month follow-up, $6 \mathrm{MW}$ distance improved by $60.3 \pm 54.1$ meters in the treated group as compared to no improvement in the control group $(0.4 \pm 185.7$ meters; $\mathrm{P}=\mathrm{ns})$. In the control group, $28.6 \%$ experienced worsening of heart failure status (4/14), while $14.3 \%$ experienced an improvement in NYHA classification (2/14). In the myoblast-treatment arm, one patient experienced a deterioration in NYHA classification (8.0\%), whereas five patients improved one or two classes (20.0\%; $\mathrm{P}=0.06$ ). However, therapy did not improve global LVEF measured by MUGA at 6-month follow-up.

Conclusions: These data indicate that implantation of myoblasts in patients with HF is feasible, appears to be safe and may provide symptomatic relief, though no significant effect was detected on global LVEF.
\end{abstract}

\footnotetext{
* Corresponding author: Thoraxcenter Rotterdam, Erasmus Medical Center, Room Ee2389A, 's Gravendijkwal 230, PO Box 2040, 3000 CA Rotterdam, The Netherlands

E-mail: h.duckers@erasmusmc.nl

(c) Europa Edition 2011. All rights reserved.
} 


\section{Introduction}

Heart failure (HF) constitutes a growing patient population, in large part due to advances in percutaneous revascularisation procedures, optimised pharmacotherapeutic guidelines and HF care, which contribute to an ageing population. An estimated five million patients in the US suffer from clinically manifested HF and account for the approximately one million hospital admissions per year. ${ }^{1}$ This profound socio-economic burden merits the exploration of novel therapeutic strategies for end-stage HF patients. Cell therapy may provide a new option in the treatment of chronic left ventricular dysfunction.

Skeletal myoblasts (SkMs) are an easily accessible source of autologous precursor cells committed to a myogenic, functionally contractile phenotype. Moreover, SkMs can be expanded extensively in vitro to therapeutic relevant dosages from limited muscle biopsies. SkMs are resistant to ischaemia, inflammatory conditions and oxidative stress, and are able to form new myotubes in scarred myocardium, which has been shown to contribute to the function of damaged myocardium in large animal infarction models. ${ }^{2}$

In 2003, Menasche et al extended these observations to the treatment of CAD patients with congestive HF undergoing surgical revascularisation. ${ }^{3}$ We previously demonstrated the feasibility of percutaneous autologous SkM transplantation following endoventricular NOGA mapping and targeted intramyocardial injection into the scar areas of chronically infarcted myocardium. ${ }^{4}$ We have now taken these studies further with the SEISMIC study, which was designed as a multicentre, prospective, controlled, randomised study to assess the safety and feasibility of percutaneous intramyocardial transplantation of autologous SkMs and to explore the efficacy in the treatment of congestive heart failure patients. Study patients were stringently monitored for arrhythmias possibly associated with cell therapy, and further analysed using exercise testing, NYHA classification of HF, Minnesota Living With Heart Failure Questionnaire (MLWHFQ) and MUGA scan analysis of global LVEF at six months.

\section{Methods}

\section{Study design}

SEISMIC is a phase lla, prospective, open-label, randomised study performed at 13 sites in six European countries to assess the safety, feasibility and cardiovascular effects of endoventricular autologous SkM implantation by a catheter delivery system (MyoCell ${ }^{\oplus}$ and MyoCath ${ }^{\mathrm{TM}}$; Bioheart Inc., Sunrise, FL, USA) in congestive HF patients. Patients were randomised 2:1 to SkM cell therapy versus optimal HF pharmacotherapy. All patients received prophylactic treatment with amiodarone, four weeks prior to cell transplantation for up to four weeks after SkM transplantation to minimise the risk of ventricular arrhythmias (200 mg qd). Subjects assigned to the cell treatment group received a skeletal muscle biopsy for SkM isolation and culture. Myoblasts were intramyocardially injected into akinetic segments under fluoroscopic guidance. Clinical evaluations were scheduled at baseline, 1, 3, and 6 months.

The primary safety endpoint was defined as the proportion of patients experiencing serious adverse events (SAEs) at three and six months follow-up. Serious adverse events were defined as: a. any adverse event that was fatal or life-threatening, b. led to prolonged or required hospitalisation, c. resulted in any major medical intervention or $d$. any sustained arrhythmia ( $>30$ seconds). SAEs were adjudicated by an independent DSMB committee. The secondary safety endpoints included deviations of routine clinical laboratory tests, 12-lead ECG recording, and the occurrence of ventricular arrhythmias on 24 hour Holter monitoring. An additional secondary safety objective was to investigate the safety of the MyoCath ${ }^{\mathrm{TM}}$ catheter to percutaneously deliver the cellular graft. The primary efficacy endpoint comprised the effect on global left ventricular ejection fraction (LVEF) at three and six months as assessed by a MUGA scan. Secondary efficacy endpoints included the change in six-minute walk (6MW) distance. The overall condition of the patients was assessed by NYHA classification of $\mathrm{HF}$, and the Minnesota Living With Heart Failure Questionnaire (MLWHFQ), frequency and duration of hospitalisations, need for medical treatment, and patient survival. Clinical parameters and analyses of functional outcome were collected and analysed by an independent clinical research organisation and independent core laboratories respectively, all blinded to the treatment of the individual patients.

\section{Patient eligibility: in/exclusion criteria}

The study intended to enrol 46 patients, randomised 2:1 to the treatment arm and the control arm. Patients were eligible for study enrolment if they had a diagnosis of ischaemic cardiomyopathy with clinically manifest HF, NYHA classification II-III, and a global left ventricular ejection fraction between 20 and $45 \%$, as measured by MUGA. Qualified patients had a defined akinetic region confirmed by either left ventricular angiography or echocardiography. Optimal pharmacological therapy was initiated at least two months prior to screening, whereas an ICD was required to be fitted at least six months prior to randomisation.

Exclusion criteria included $\mathrm{CABG}$ or $\mathrm{PCl}$ within three months prior to cell implantation, HF secondary to valvular disease, ventricular thrombus or significant aortic stenosis.

\section{Quadriceps muscle biopsy and cell culture expansion and transplantation}

Autologous SkMs were isolated and cultured from approximately 10 grams of a skeletal muscle biopsy derived from the quadriceps or gastronemicus muscle as previously described. ${ }^{4}$ The SkM grafts were required to contain at least $50 \% \mathrm{SkMs}$, as determined by flow cytometric analysis of CD56 and desmin immunostaining. The myoblast cellular graft was intramyocardially injected into the targeted treatment region utilising the MyoCath ${ }^{T M}$ catheter delivery system under fluoroscopic guidance as previously described. ${ }^{5}$ The treatment group received a dose in the range of 150-800 million cells, dependent on the size of ventricular scar tissue to be treated. Since the cell concentration was maintained at 50 million cells $/ \mathrm{mL}$, the final number of injections varied per patient. The volume per injection was $0.5 \mathrm{~mL}$, whereas a maximum number of 32 injections was allowed per implantation procedure. 


\section{Holter monitoring of arrhythmia}

To distinguish a possible pro-arrhythmic effect of intramyocardial cell therapy, patients were assessed for pre-existing arrhythmias using weekly 24-hour Holter monitoring, initiated at least six weeks prior to MyoCell ${ }^{\circledR}$ implantation. Following intramyocardial SkM transplantation, the patient was monitored by continuous telemetry monitoring for a minimum of 72 hours after the procedure, followed by 48 hour Holter monitoring. From the one-week follow-up period onward, patients were subjected to 48 hour Holter monitoring every week, for up to the four weeks post-transplantation, and at three and six months. Any abnormal finding(s) or firing identified by the ICD were assessed immediately by the investigative team at the clinical site.

\section{Statistical analysis}

As SEISMIC was designed to be an exploratory randomised phase Ila study to primarily assess the safety and feasibility of the percutaneous MyoCell ${ }^{\circledR}$ transplantation procedure, and secondarily to provide preliminary data regarding the efficacy of intramyocardial SkM transplantation, no formal power calculations were performed. Two-tailed t-tests were performed for all analyses and confidence intervals are presented with 95\% degree of confidence. A two-way ANOVA with repeated measures was used for the comparison of NYHA classification of HF and MUGA left ventricular ejection fraction. All statistical tests used a significance level of $\alpha \leq 0.05$.

The parametric assumption of homogeneity of variance for the two treatment groups was checked using the F-test and normality of the two samples was explored using the Wilk-Shapiro test for normality. To support a non-parametric analysis, a 95\% confidence for the difference in medians is presented. For analysis of SAEs, a Chisquare test (with continuity correction) was used, presented with a 95\% confidence interval for the difference in proportions. If expected cell frequencies were small $(\leq 5)$, then Fisher's exact test was used instead.

\section{Results}

\section{Patient cohort and baseline evaluation}

Between October 2005 and May 2007, a total of 62 patients were screened for enrolment in the study at 13 European sites. Of the 62 subjects, 12 failed screening, and were excluded. Three subjects voluntarily withdrew during the screening process. The remaining 47 patients were enrolled and randomised in a 2:1 fashion. Thirty-one patients were randomised to receive SkM transplantation and 16 were randomised to the control group. Of the 31 patients randomised to the MyoCell ${ }^{\circledR}$ therapy group, five patients voluntarily withdrew from the study following changes in the German regulations concerning biopsies. Two patients withdrew from the control group upon knowledge of their randomisation allocation. Thus, a total of 40 patients entered the study, 26 of whom entered the SkM cell therapy arm, and 14 entering the control arm (Figure 1). Baseline clinical characteristics and demographics of the two patient groups are listed in Table 1.

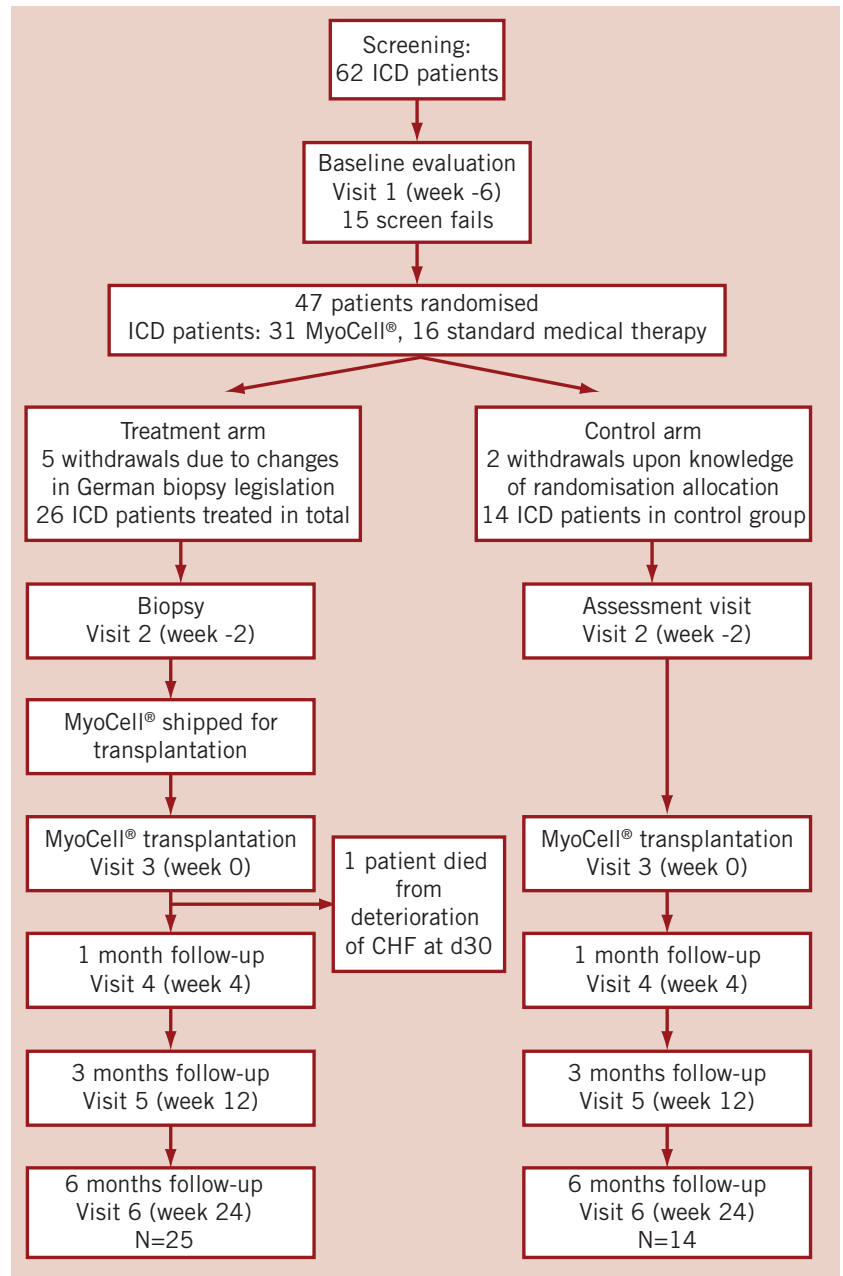

Figure 1. Flow chart of the SEISMIC trial. From the 62 patients that were initially screened, 47 were randomised due to screen failure of 15 patients. Five German patients that were allocated in the treatment arm were withdrawn due to changed biopsy legislation. In the control arm, two patients withdrew upon knowledge of their randomisation allocation. 26 patients entered the treatment arm of the study and 14 patients entered the control arm.

\section{Muscle biopsy and cell culturing}

All procedures were well tolerated and uneventful, resulting in a biopsy of $8.6 \pm 4.2 \mathrm{~g}$. Two patients developed a post-procedural haematoma that resolved without sequelae. On average, $920 \pm 302 \times 10^{6}$ myoblasts were generated with a viability of $96.7 \pm 2.1 \%$. In all cultured cellular grafts, the CD56+ cell content was at least $50 \%$ at the time point of cell harvest $(89.0 \pm 13.2 \%$ desmin+; 57.2\% $\pm 23.9 \%$ CD56+).

\section{Transplantation procedure}

The transplantation procedure was successful in 26/27 patients (96.3\%). Patients scheduled for myoblast transplantation received an average dose of $596 \pm 194 \times 10^{6}$ cells in $26 \pm 7$ injections (range $12-35$; in 18.8 $\pm 6.8 \mathrm{cc}$ [range $9-30 \mathrm{cc}$ ]).

In one patient, the implantation procedure was complicated by a dissection of the left main coronary artery, the ascending and descending aorta, upon dislocation of the MyoCath ${ }^{\mathrm{TM}}$ injection 
Table 1. Baseline patient demographics and clinical characteristics.

\begin{tabular}{|c|c|c|c|}
\hline Patient parameter measured & $\begin{array}{l}\text { MyoCell }{ }^{\circledR} \\
(\mathrm{n}=26)\end{array}$ & $\begin{array}{l}\text { Control } \\
(n=14)\end{array}$ & $p$-value ${ }^{1}$ \\
\hline \multicolumn{4}{|l|}{ Sex } \\
\hline Male & $92 \%(24 / 26)$ & $71 \%(10 / 14)$ & 0.16 \\
\hline Female & $8 \%(2 / 26)$ & $29 \%(4 / 14)$ & \\
\hline \multicolumn{4}{|l|}{ Age (years) } \\
\hline mean $\pm S D$ & $59.2 \pm 8.64$ & $61.9 \pm 8.38$ & 0.35 \\
\hline$(\min -\max )$ & $(32-72)$ & $(44-75)$ & \\
\hline \multicolumn{4}{|l|}{ Pharmacotherapy } \\
\hline Beta blockade & $89 \%(23 / 26)$ & $79 \%(11 / 14)$ & 0.64 \\
\hline ACE inhibition or ARB & $96 \%(25 / 26)$ & $100 \%(14 / 14)$ & 1.0 \\
\hline Diuretics & $69 \%(18 / 26)$ & $71 \%(10 / 14)$ & 1.0 \\
\hline Anti-arrhythmic drugs & $100 \%(26 / 26)$ & $100 \%(14 / 14)$ & 1.0 \\
\hline Coumarin derivative & $35 \%(9 / 26)$ & $36 \%(5 / 14)$ & 1.0 \\
\hline \multicolumn{4}{|l|}{ Previous cardiovascular history } \\
\hline Myocardial infarction & $100 \%(26 / 26)$ & $100 \%(14 / 14)$ & 1.0 \\
\hline Ventricular arrhythmia & $73 \%(19 / 26)$ & $71 \%(10 / 14)$ & 1.0 \\
\hline $\begin{array}{l}\text { Aborted sudden cardiac death } \\
\text { OOHA }\end{array}$ & $4 \%(1 / 26)$ & $0 \%(0 / 14)$ & 1.0 \\
\hline $\begin{array}{l}\text { ICD implanted prior } \\
\text { to study requirement }\end{array}$ & $96 \%(25 / 26)$ & $100 \%(14 / 14)$ & 1.0 \\
\hline Previous PTCA & $27 \%(7 / 26)$ & $14 \%(2 / 14)$ & 0.45 \\
\hline Previous CABG & $23 \%(6 / 26)$ & $21 \%(3 / 14)$ & 0.44 \\
\hline Diabetes mellitus & $31 \%(8 / 26)$ & $14 \%(2 / 14)$ & 0.45 \\
\hline CVA/TIA & $0 \%(0 / 26)$ & $14 \%(2 / 14)$ & 0.14 \\
\hline LVEF (MUGA) & $35 \pm 9 \%$ & $33 \pm 11 \%$ & 0.54 \\
\hline NYHA & & & 0.32 \\
\hline NYHA I & $0 \%(0 / 26)$ & $0 \%(0 / 14)$ & \\
\hline NYHA II & $62 \%(16 / 26)$ & $79 \%(11 / 14)$ & \\
\hline NYHA III & $38 \%(10 / 26)$ & $21 \%(3 / 14)$ & \\
\hline NYHA IV & $0 \%(0 / 26)$ & $0 \%(0 / 14)$ & \\
\hline MLWHFQ (QOL) & $40.4 \pm 21.9$ & $45.1 \pm 29.1$ & 0.32 \\
\hline
\end{tabular}

Numbers are\% (counts/available field sample size) or mean \pm 1 Standard Deviation. ${ }^{1} P$-value is from a Fisher's exact test; SD standard deviation; ICD: implantable cardioverter defribillator; MI: myocardial infarction; CABG: coronary artery bypass graft; PTCA: percutaneous transluminal coronary angioplasty; MLWHFQ: Minnesota Living With Heart Failure Questionnaire catheter during intramyocardial injection. The transplant procedure was ceased after seven injections, with the coronary dissection successfully treated by direct stent implantation, resulting in recovery of coronary flow and stabilisation of the patient. The aortic dissection was treated conservatively, and the patient recovered without persistent myocardial or neurological sequelae $\left(\mathrm{CK}_{\max }<300\right)$. This patient was excluded from the study resulting in 26 patients in total in the treatment arm.

\section{All MACCE}

An overview of the adjudicated SAEs is summarised in Table 2. No unanticipated adverse events attributable to the SkM therapy were reported, whereas no difference in the incidence of SAEs was observed in the cell therapy and control arm. A total of 21 SAEs occurred in 11 out of 26 patients subjected to SkM transplantation (42\%), as opposed to 23 SAEs occurring in five out of the 14 control patients (36\%; $P=0.68$ ). Specifically, 20 cardiovascular SAEs occurred in 11 patients treated with SkM (11/26; 42\%), whereas 18 cardiovascular events occurred in four patients in the control $\operatorname{arm}(4 / 14 ; 29 \% ; P=0.39)$.
Table 2. Serious adverse events \& adverse events at 6-months.

\begin{tabular}{|c|c|c|c|c|c|}
\hline Serious adverse event & $\begin{array}{c}\text { MyoCell }{ }^{\circledR} \\
(\mathrm{n}=26)\end{array}$ & & $\begin{array}{l}\text { Control } \\
(n=14)\end{array}$ & & $p$-value ${ }^{1}$ \\
\hline & & pisodes & & Episodes & \\
\hline Cardiovascular & & & & & \\
\hline Bradycardia & $3.8 \%(1 / 26)$ & 1 & $14.3 \%(2 / 14)$ & 2 & 0.27 \\
\hline Ventricular fibrillation & $3.8 \%(1 / 26)$ & 1 & $0.0 \%(0 / 14)$ & 0 & 1.0 \\
\hline Ventricular tachycardia & $23.1 \%(6 / 26)$ & 11 & $21.4 \%(3 / 14)$ & 12 & 1.0 \\
\hline Idioventricular rhythm & $0.0 \%(0 / 26)$ & 0 & $14.3 \%(2 / 14)$ & 2 & 0.12 \\
\hline Non-sustained VT with & & & & & \\
\hline hospitalisation & $3.8 \%(1 / 26)$ & 1 & $0.0 \%(0 / 14)$ & 0 & 1.0 \\
\hline Worsening of $\mathrm{CHF}$ & $3.8 \%(1 / 26)$ & 1 & $7.1 \%(1 / 14)$ & 1 & 1.0 \\
\hline Hypotension & $0.0 \%(0 / 26)$ & 0 & $7.1 \%(1 / 14)$ & 1 & 0.35 \\
\hline Pulmonary oedema & $3.8 \%(1 / 26)$ & 1 & $0.0 \%(0 / 14)$ & 0 & 1.0 \\
\hline Aorta dissection & $3.8 \%(1 / 26)$ & 1 & $0.0 \%(0 / 14)$ & 0 & 1.0 \\
\hline Pericarditis & $3.8 \%(1 / 26)$ & 1 & $0.0 \%(0 / 14)$ & 0 & 1.0 \\
\hline Gastrointestinal & & & & & \\
\hline Diverticulitis & $0.0 \%(0 / 26)$ & 0 & $7.1 \%(1 / 14)$ & 1 & 0.35 \\
\hline Gastroenteritis & $3.8 \%(1 / 26)$ & 1 & $0.0 \%(0 / 14)$ & 0 & \\
\hline Renal dysfunction & $0.0 \%(0 / 26)$ & 0 & $14.3 \%(2 / 14)$ & 2 & 0.12 \\
\hline Pulmonary & & & & & \\
\hline Exacerbation COPD & $3.8 \%(1 / 26)$ & 1 & $0.0 \%(0 / 14)$ & 0 & 1.0 \\
\hline Infection & & & & & \\
\hline Urinary tract & $0.0 \%(0 / 26)$ & 0 & $7.1 \%(1 / 14)$ & 1 & 0.35 \\
\hline MRSA & $0.0 \%(0 / 26)$ & 0 & $7.1 \%(1 / 14)$ & 1 & 0.35 \\
\hline Death & $3.8 \%(1 / 26)$ & 1 & $0.0 \%(0 / 14)$ & 0 & 1.0 \\
\hline Total number of SAEs & 21 & & & 23 & \\
\hline Number of subjects & $42.0 \%(11 / 26)$ & & $36.0 \%(5 / 14)$ & 0.95 & \\
\hline Subjects with & & & & & \\
\hline cell therapy-related SAEs ${ }^{2}$ & $30.8 \%(8 / 26)$ & & & & \\
\hline $\begin{array}{l}\text { Unanticipated cell } \\
\text { therapy-related SAEs }\end{array}$ & $0.0 \%(0 / 26)$ & & & & \\
\hline
\end{tabular}

Subjects reporting a particular event more than once are only counted once using the most severe intensity for that event. ${ }^{\prime * 1}$ denotes statistical significance at the 0.05 level. ${ }^{1} P$-value is from a Fisher's exact test. ${ }^{2}$ Celltherapy-related events are those events where relationship is possible/ probable/definite to MyoCell ${ }^{\circledR}$, related to MyoCath ${ }^{\text {TM }}$ device or related to implant procedure. ${ }^{3}$ Unanticipated events have been identified through a medical review of all serious adverse event information by a medically qualified individual.

One patient in the myoblast therapy group died during the study period (3.8\%), whereas no deaths occurred in the control patient group during study follow-up. This particular patient had a previous medical history of ischaemic cardiomyopathy, impaired renal and hepatic function, ventricular arrhythmias and ICD implantation four years prior to enrolment. The MyoCell ${ }^{\circledR}$ implantation of 625 million cells was uneventful. From day five to day 10 , post-implantation, the patient experienced several episodes of monomorphic, sustained VT, which were successfully terminated via anti-tachycardia pacing. In addition to beta-blockers and amiodarone, flecainide was initiated, rendering the patient bradycardic and ICD/pacemaker dependent. At 27 days, the patient deteriorated with the clinical manifestation of progressive $\mathrm{HF}$, which progressed into multi-organ failure and death at 30 days post cell transplantation. The local investigator and two independent cardiologists considered the cause of death to be progression of terminal HF, with secondary multiple organ failure, possibly provoked by flecainide with acute 
renal failure. Alternatively, multiple episodes of VT not manageable with anti-arrhythmic therapy may have initiated the progressive deterioration of cardiac and renal function. Upon autopsy, no evidence of thrombus formation or other signs of inflammation could be detected at the site of intracardiac cell injection by macroscopic or microscopic analysis. In the examined samples, the pathologist did not find clusters of injected SkMs or newly formed myotubes. A relation of this SAE with the MyoCell ${ }^{\circledR}$ implantation, however, cannot be excluded.

\section{Ventricular arrhythmias}

All patients in the myoblast and control arm received oral amiodarone therapy at the time of cell transplantation. In the control patient group, three patients experienced one or more episodes of sustained VT (3/14 pts; $21 \%$ ), as opposed to seven patients in the myoblast treatment group $(6 / 26 ; 23 \%)$. Further, the 14 control patients experienced a total 12 periods of sustained VT, as opposed to 11 events in the 26 cell therapy patients. One of these patients in the treatment group experienced two episodes of sustained VT that required ablation of the ventricular focus localised at the myocardial scar area where SkM were injected.

Previously, ventricular tachyarrhythmias were mainly reported in the first four weeks following myoblast transfer. However, in the control and the SkM group at hand, the number of patients and the frequency of arrhythmic events were equally distributed among the six month observation period and did not appear to correlate with the dose of SkMs administered (data not shown). Thus, there was neither a time, nor a dose relationship between tachyarrhythmic events and the index procedure, as compared to the untreated control group.

\section{Progression of congestive heart failure}

Other cardiovascular SAEs included the worsening of congestive HF of subjects requiring hospitalisation during the six month observation period. This occurred on three occasions in the 26 patients of the SkM therapy group (3/26 patients; $11.5 \%$ ) as compared to one admission in the matched control group (7.1\%). A Kaplan-Meier survival curve for the time to onset of the first SAE or death is shown in Figure 2.

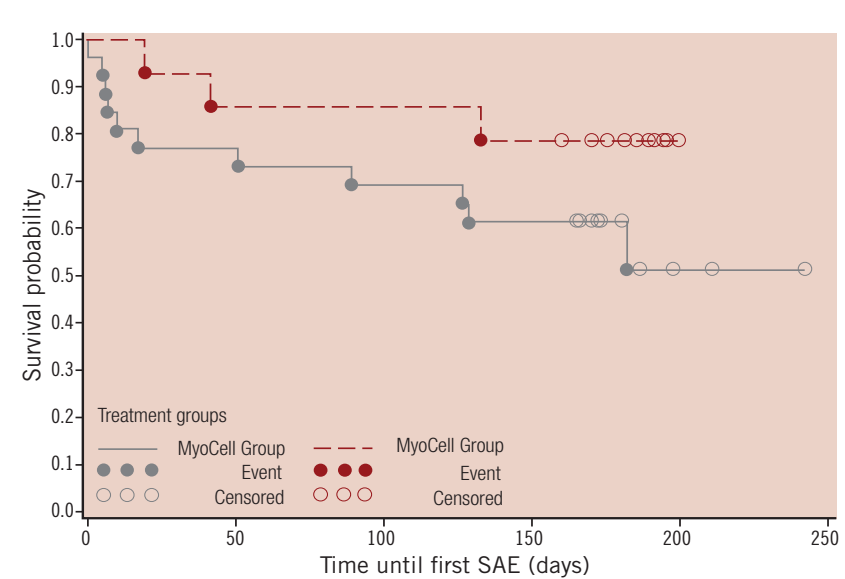

Figure 2. Kaplan-Meier survival curve for time to onset of first SAE or death from time of treatment (MyoCel/ $\left.{ }^{\oplus}\right) /$ Week O Visit (Control).

\section{Global ventricular ejection fraction by MUGA-scan}

The average baseline LVEF in the myoblast treatment arm was $32.3 \pm 9.1 \%(n=24)$ as compared to $32.6 \pm 11.1 \%$ in the control arm ( $n=14$; Figure 3). One patient in the cell therapy group died prior to the three month MUGA evaluation and was omitted from the analysis. Sequential data were obtained in 24 of the 26 patients in the cell therapy arm and in all 14 patients in the control arm. At six months follow-up, global LVEF was $32.5 \pm 8.0 \%$ in the control patient group, and $31.5 \pm 11.1 \%$ in the SkM-treated group (Figure 3; $\mathrm{P}=0.46$ ).

\section{NYHA heart failure classification and the Minnesota Living With Heart Failure Questionnaire}

During the 6-month study follow-up, the NYHA HF classification deteriorated in $28.6 \%$ in the control arm (4/14), whereas $57.1 \%$ remained stable. In the active cell therapy arm, only $8 \%$ showed a deterioration of NYHA classification, whereas the majority of patients even improved in $20.0 \%$ or remained unchanged $(72.0 \%$ ) as compared to baseline (Figure $4 \mathrm{~A} ; \mathrm{P}=0.06$ ). Thus, a trend towards improvement of NYHA classification of heart failure was noted in the SkM-treated patients.

The number of patients that experienced improvement of MLWHFQ over the six months follow-up increased from $33.3 \%$ to $52.2 \%$ with cell therapy, whereas the patients who did not notice a change in their quality of life was reduced from $41.7 \%$ to $8.7 \%$. However, the mean MLWHFQ score was not significantly different between both arms (Figure 4B; $P=0.99$ ).

\section{6 -minute walking test}

At baseline, the average distance walked in the six-minute walking (6MW) test for patients randomised to the cell therapy arm was lower than in the control arm $(404.6 \pm 94.8$ meters [ $n=23]$ vs. $442.5 \pm 154.3$ meters $[n=13]$ ), although this difference was not statistically significant.
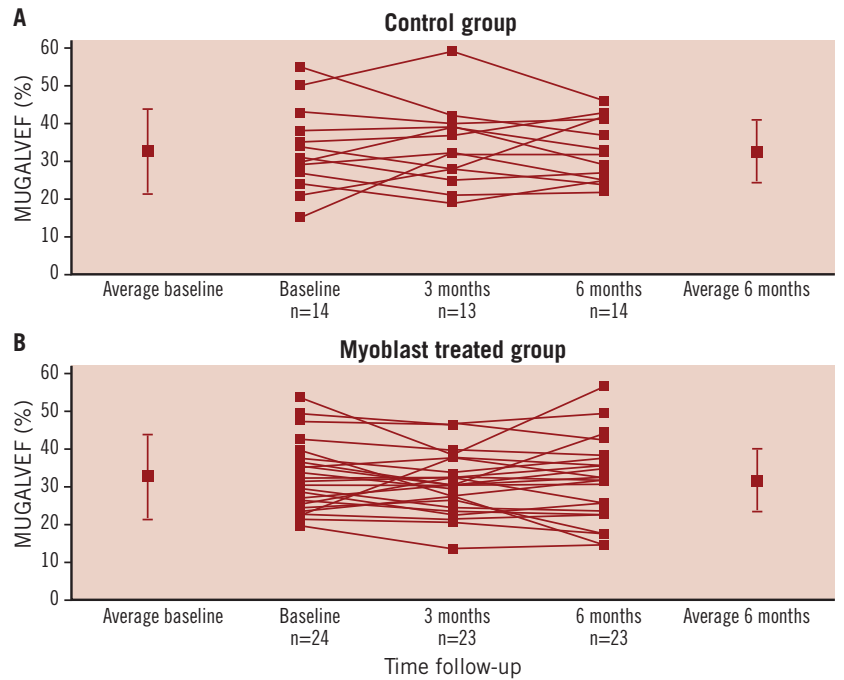

Figure 3. Individual six month change in ejection fraction from baseline to six month follow-up in the control group (A) and the skeletal myoblast treated group (B) as measured by MUGA scan. The mean data and standard deviation are denoted separately. No significant change in LVEF was found between the myoblast treated group and control. 

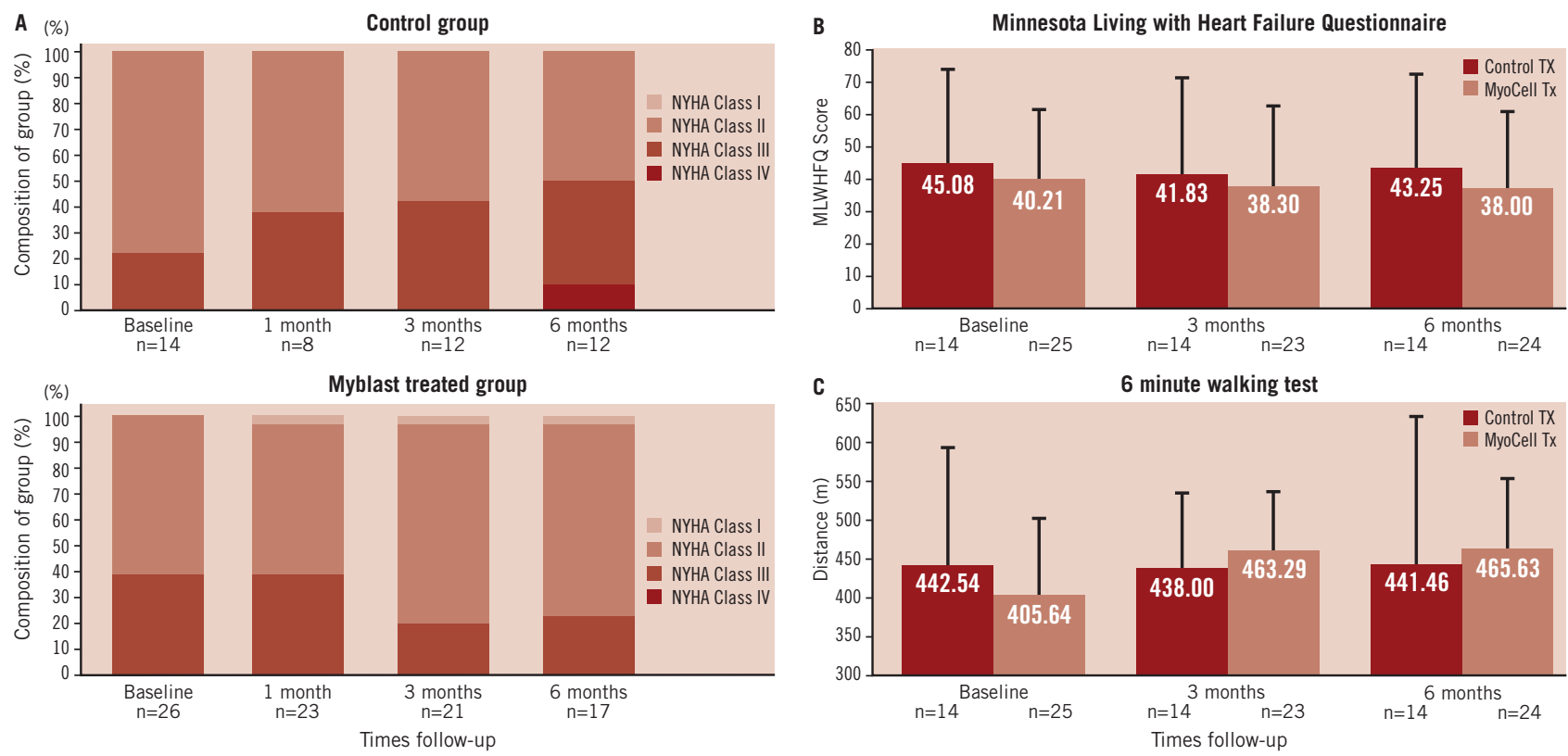

Figure 4. Six month change from baseline in NYHA Class (A), Minnesota Living With Heart Failure Questionnaire (B) and exercise tolerance (C). NYHA Class deteriorated in $28.6 \%$ of the cases in the control arm, whereas $57.1 \%$ remained unchanged. In the active cell therapy arm NYHA classification either improved or remained unchanged (20.0\% and $72.0 \%$ respectively, $P=0.06$ ). Also in the Minnesota Living With Heart Failure Questionnaire a trend to improvement was seen in treated patients (light red) vs. control patients (dark red), but this difference was not statistically significant $(p=0.99)$. Exercise tolerance was measured by six minute walking test in treated (dark red) and control (light red) patients. Although a trend to an increased exercise tolerance was seen, the difference between the treated and control group was not significant ( $p=0.49)$.

The distance walked in the control group remained virtually unchanged at six month follow-up ( $+0.4 \pm 185$ meters). In contrast, the distance walked in the treated group improved by +60.3 meters to $465.6 \pm 89.5$ meters (Figure $4 \mathrm{C}$ : $P=0.49$ ). The walking distance improved or remained unchanged in $87 \%$ of the myoblast-treated patients, as opposed to $30.8 \%$ in the control arm. In contrast, exercise tolerance deteriorated in $69.2 \%$ in the control arm as opposed to $13.0 \%$ in the cell therapy arm.

\section{Discussion}

The rationale for the investigation of cell therapy for $\mathrm{HF}$ is based on the assumption that development of HF is linked to irreversible loss of contractile myocardium to below a certain threshold and that myocardial function can be improved by replacing lost muscle with cells that graft to, and function as native myocardium. In experimental models, SkMs have been transplanted into infarcted cardiac muscle and non-ischaemic, globally dilated myocardium, and have been shown to subsequently develop into elongated, striated muscle cells which retain characteristics of both skeletal muscle and cardiac cells. ${ }^{6-9}$ In these models, intramyocardial myoblast engraftment has been associated with a dose-dependent improvement in LV function. Additionally, SkMs are able to release trophic growth factors that exert an anti-fibrotic effect and enhance cardiac performance and myocardial perfusion through the stimulation of local angiogenesis. , $^{10}$

The safety concerns regarding endomyocardial SkM therapy predominantly relate to a possible pro-arrhythmogenic effect of the intramyocardial injection technique and the SkM graft. Irrespective of whether cells were transferred as an adjunct to revascularisation surgery, or by percutaneous endomyocardial injection, SkM therapy was previously associated in a small number of patients with recurrent premature ventricular systoles and (non)sustained ventricular arrhythmias. This was seen particularly during the first 30 days after transplantation. ${ }^{3}$

In the SEISMIC trial, no significant difference was detected between treatment groups, frequency of ventricular arrhythmic events or the percentage of patients with documented ventricular tachyarrhythmia via ICD interrogation (Table 2). SkM transplantation was neither associated with an increased incidence of ventricular tachyarrhythmias, nor in the absolute number of episodes in the treatment group. In the control patient group, $21 \%$ of the patients experienced one or more episodes of serious ventricular tachyarrhythmias (a total of 12 episodes of VTNF), as opposed to $27 \%$ in the myoblast treatment group (total of 12 episodes of VTNF). Ablation of a ventricular focus was indicated in one patient due to refractory tachyarrhythmias at 10 and 16 weeks following SkM transplantation. Although a causal relationship between these tachyarrhythmic events and the SkM transplantation can not be excluded, the time interval between the cell injection procedure and the onset of VTs renders a relationship less probable. The SkMs were injected in scar tissue that by itself could give rise to ventricular tachyarrhythmias. In another patient, tachyarrhythmias at five days following SkM therapy may have provoked deterioration and progression into terminal HF. Even though this particular patient was known with multiple episodes of VT before cell injection and an ICD was implanted four years earlier, causality between the intramyocardial injections and the VTs cannot be ruled out. Although the current study did not suggest a time and dose 
correlation between tachyarrhythmic events and the index procedure, a pro-arrhythmic effect associated with the myoblast transplantation procedure in these two patients cannot be excluded. However, the current data provide a basis to further explore the therapeutic value of SkM transplantation in larger randomised and placebo-controlled trials with prophylactic amiodarone and ICD implantation.

In various pre-clinical animal models, SkM therapy has been shown to reduce myocardial remodelling, impede ECM deposition and cardiomyocyte apoptosis and replenish lost cardiomyocytes in the chronic scar by introducing new contractile myocytes into the region of dysfunction. ${ }^{2,6,11}$ Immunohistological and patch clamp studies of myoblasts have shown effective re-population of the fibrotic tissue and transition from a "fast" to a "slow twitch" phenotype upon cardiac transplantation eventually leading to restoration of contractile function. Skeletal myoblast therapy is to date the only regenerative cell therapy approach primarily aimed at neomyogenesis, rather than neoangiogenesis in the myocardial scar, in post AMI heart failure patients.

In the MAGIC trial, intramyocardial SkM transplantation as an adjunctive to CABG was studied in patients with ischaemic HF with an ejection fraction under 35\%. ${ }^{12}$ Although SkM transfer failed to augment the restoration of regional and global LV function as determined by echocardiography in these patients undergoing surgical revascularisation, an improvement in LV dimensions was observed in the high dose cell group. In contrast, in the open-label, dose-escalating, randomised MYOHEART and CAUSMIC trials, trends towards beneficial clinical and functional effects of myoblast therapy were reported in patients with ischaemic heart failure following endoventricular injection in respectively 20 and 12 patients. $^{13}$

In the current study, SkM therapy appeared to attenuate further deterioration of NYHA classification of HF, with an improvement in exercise tolerance in a 6MWT, as opposed to a lack of improvement in the control arm. However, this could not be substantiated by an improvement in global LVEF, quantified by nuclear ventriculography. The lack of a clear effect on global LVEF can be partly explained by our previous studies in preclinical and clinical myoblast transplantation, which suggested that on short term, akinesia in scarred or hibernating myocardium might be compensated by hyper-contractility in remote myocardial segments to preserve global LV function. A possible beneficial effect of stem cell therapy leading to improved local wall motion in the afflicted wall segment may be concealed by normalisation of the compensatory hypercontractility in remote wall segments, resulting in a modest overall effect on global cardiac function. Moreover, the long term follow-up of patients with LV dysfunction by conventional echocardiographic or scintigraphic LVEF quantification is based on the presumption of a comparable haemodynamic state of patients at baseline and follow-up and cannot be corrected for change in LV loading. As myocardial cell therapy aims to improve the contractile function and haemodynamic state, comparing LVEF with the assumption of static LV loading may underestimate the beneficial effect on LV function. Invasive haemodynamic analysis of systolic and diastolic LV function by the analysis of pressure volume-loop relations under dynamic LV loading (LV unloading by use of gradual preload reduction), can however provide load-independent indices of global and intrinsic systolic and diastolic function. Indeed, in a long term follow-up of patients that received endoventricular myoblast injection, pressure-volume relation analysis provided proof of improved systolic and diastolic function at six and 12-month followup, whereas nuclear radiography and magnetic resonance imaging failed to reflect this improvement. ${ }^{14}$

Although SEISMIC suggests that the use of SkM therapy is safe, the mixed results on functional efficacy endpoints, coupled with the general underpowering of the study, render any interpretation inconclusive. Further, since the current trial was an open-label study, a potential effect of patient bias on the positive effects on clinical endpoints cannot be ruled out, and underscores the necessity to conduct appropriately powered clinical studies to evaluate cell therapy, blinded to both clinical staff and patients, with outsourcing of study end point analyses to independent core laboratories. Hence, the recently initiated MARVEL study seeks to explore the therapeutic efficacy of NOGA guided endomyocardial myoblast therapy in chronic LV dysfunction in a prospective, randomised, double-blinded, placebo-controlled study enrolling 390 patients in up to 35 US and EU clinical centres.

\section{Conclusion}

The current study sought to define the safety and feasibility of percutaneous intramyocardial transplantation of autologous SkM in 40 patients with chronic HF with an ischaemic origin. One could argue whether this would be the most appropriate target population to provide proof of principle of a neomyogenesis therapy concept, since end-stage heart failure patients may lack the plasticity to respond to reverse remodelling by stem cell therapy. The current study failed to detect a benefit on global LV function, possibly because it was outweighed by the advanced LV dysfunction in end-stage HF or fell beyond the detection limits of radionuclide ventriculography. In addition, the small sample size in a heterogeneous population could have further concealed a possible beneficial effect on global LVEF. Since the studies in the chronic HF stem cell therapy field are still at a very preliminary stage, they are exploratory in nature, and thus by virtue, underpowered. Therefore a true evaluation of efficacy and safety cannot be guaranteed by the study at hand, and should be validated in forthcoming phase II/III studies. However, the current study does provide encouraging feasibility data regarding the endomyocardial stem cell treatment, and suggests some clinical benefit of SkM therapy in HF patients.

\section{Funding sources}

The study was supported by Bioheart Inc, Sunrise, Florida, USA.

\section{References}

1. Hunt SA. ACC/AHA 2005 guideline update for the diagnosis and management of chronic heart failure in the adult. J Am Coll Cardiol. 2005;46:e1-82.

2. Menasche P. Skeletal myoblasts and cardiac repair. J Mol Cell Cardiol. 2008;45:545-53. 
3. Menasche $P$, Hagege AA, Vilquin JT, Desnos $M$, Abergel $E$, Pouzet B, Bel A, Sarateanu S, Scorsin M, Schwartz K, Bruneval P, Benbunan M, Marolleau JP, Duboc D. Autologous skeletal myoblast transplantation for severe postinfarction left ventricular dysfunction. J Am Coll Cardiol. 2003;41:1078-83.

4. Smits PC, van Geuns RJ, Poldermans D, Bountioukos M, Onderwater EE, Lee CH, Maat AP, Serruys PW. Catheter-based intramyocardial injection of autologous skeletal myoblasts as a primary treatment of ischemic heart failure: clinical experience with six-month follow-up. J Am Coll Cardiol. 2003;42:2063-9.

5. Ince $H$, Petzsch M, Rehders TC, Chatterjee T, Nienaber CA. Transcatheter transplantation of autologous skeletal myoblasts in postinfarction patients with severe left ventricular dysfunction. J Endovasc Ther. 2004;11:695-704.

6. Dowell JD, Rubart M, Pasumarthi KB, Soonpaa MH, Field LJ. Myocyte and myogenic stem cell transplantation in the heart. Cardiovasc Res. 2003;58:336-50.

7. van den Bos EJ, Thompson RB, Wagner A, Mahrholdt $H$, Morimoto Y, Thomson LE, Wang LH, Duncker DJ, Judd RM, Taylor DA. Functional assessment of myoblast transplantation for cardiac repair with magnetic resonance imaging. Eur J Heart Fail. 2005;7:435-43.

8. McConnell PI, del Rio CL, Jacoby DB, Pavlicova M, Kwiatkowski P, Zawadzka A, Dinsmore JH, Astra L, Wisel S, Michler RE. Correlation of autologous skeletal myoblast survival with changes in left ventricular remodeling in dilated ischemic heart failure. J Thorac Cardiovasc Surg. 2005;130:1001.
9. Maurel A, Azarnoush K, Sabbah L, Vignier N, Le Lorc'h M, Mandet C, Bissery A, Garcin I, Carrion C, Fiszman M, Bruneval P, Hagege A, Carpentier A, Vilquin JT, Menasché P. Can cold or heat shock improve skeletal myoblast engraftment in infarcted myocardium? Transplantation. 2005;80:660-5.

10. Formigli L, Perna AM, Meacci E, Cinci L, Margheri M, Nistri S, Tani A, Silvertown J, Orlandini G, Porciani C, Zecchi-Orlandini S, Medin J, Bani D. Paracrine effects of transplanted myoblasts and relaxin on postinfarction heart remodelling. J Cell Mol Med. 2007;11:1087-100.

11. van den Bos EJ, Davis BH, Taylor DA. Transplantation of skeletal myoblasts for cardiac repair. J Heart Lung Transplant. 2004;23:1217-27.

12. Menasche P, Alfieri O, Janssens S, McKenna W, Reichenspurner H, Trinquart L, Vilquin JT, Marolleau JP, Seymour B, Larghero J, Lake S, Chatellier G, Solomon S, Desnos M, Hagège AA. The Myoblast Autologous Grafting in Ischemic Cardiomyopathy (MAGIC) trial. Circulation. 2008;117:1189-200.

13. Dib N, Dinsmore J, Lababidi Z, White B, Moravec S, Campbell A, Rosenbaum A, Seyedmadani K, Jaber WA, Rizenhour CS, Diethrich E. One-year follow-up of feasibility and safety of the first U.S., randomized, controlled study using 3-dimensional guided catheter-based delivery of autologous skeletal myoblasts for ischemic cardiomyopathy (CAuSMIC study). JACC Cardiovasc Interv. 2009;Jan;2:9-16.

14. Steendijk P, Smits PC, Valgimigli M, van der Giessen WJ, Onderwater EE, Serruys PW. Intramyocardial injection of skeletal myoblasts: Iong-term follow-up with pressure-volume loops. Nat Clin Pract Cardiovasc Med. 2006;3 Suppl 1:S94-100. 